\title{
Rede Social de Pessoas com Consumos Aditivos e Dependências
}

\author{
Social Network of People with Addictive Behaviors and Dependencies
}

Artigo Original | Original Article

\author{
Ana Paula Caetano, PsyM, SW (1a), Sónia Guadalupe, PhD (2b) \\ (1) Florinhas do Vouga. Aveiro, Portugal. \\ (2) Centro de Estudos da População, Economia e Sociedade (CEPESE), Centro de Investigação e Estudos de Sociologia (CIES-IUL, ISCTE), Instituto Superior Miguel Torga. Coimbra, Portugal. \\ (a) Redação, recolha dos dados, construção da base de dados, análise preliminar de dados e discussão. \\ (b) Redação, análise de dados, discussão e revisão do trabalho. \\ Autor para correspondência | Corresponding author: Ana Paula Caetano; e-mail: paula.caetano.12@gmail.com
}

\section{Palavras-Chave}

Consumos aditivos e dependências

Exclusão social

Rede social

Serviço social

Suporte social

\section{RESUMO}

Objetivos: As redes sociais desempenham um papel central na qualidade de vida da pessoa com comportamentos aditivos, determinantes, tanto na manutenção como na compensação e na reabilitação destes comportamentos. Este estudo tem como objetivo caracterizar as redes sociais de pessoas com consumos aditivos e dependências em situação ou risco de exclusão social.

Métodos: Participaram no estudo 30 sujeitos consumidores de substâncias psicoativas, com idades compreendidas entre os 24 e os 65 anos $(M \pm D P=43,03 \pm 10,10)$, a maioria do sexo masculino (80,0\%), não tendo fonte de rendimentos na maior parte $(46,7 \%)$ ou sendo apoiados por medidas de política social (53,3\%). A maioria tem como substância principal de consumo a heroína (63,3\%). Avaliámos as dimensões estrutural, funcional e relacional-contextual das suas redes com o Instrumento de Análise da Rede Social Pessoal.

Resultados: As redes dos inquiridos são constituídas, em média, por 7 elementos, predominando as relações familiares; são fragmentadas, constituídas essencialmente por membros das redes primárias, ainda que as secundárias representem cerca de $1 / 4$ dos membros. A reciprocidade e o nível de apoio social percebido são elevados, assim como o apoio emocional e informativo. Os indivíduos inquiridos, na sua maioria, refere ter membros na rede com CAD na rede social (77,0\%), relações que ocupam mais de $20,0 \%$ do tamanho das redes. Analisámos comparativamente as redes segundo privação material e de abrigo, não tendo emergido diferenças significativas $(p>0,05)$, ainda que os perfis de rede sugiram diferentes estratégias de ativação do suporte.

Conclusões: $O$ estudo confirma a importância da avaliação da rede social em pessoas com comportamentos aditivos e dependências. A planificação da intervenção social deve integrar as características da rede pertinentes para o suporte na redução do uso abusivo de substâncias e dos riscos associados, assim como para a promoção do bem-estar social e da cidadania.

\section{ABSTRACT}

Aims: Social networks play a central role in the quality of the life of persons with additive behaviors, being determinant both in the maintenance, compensation or rehabilitation of these behaviors. This study aims to characterize the social personal networks of people with additive behavior and dependencies at risk of social exclusion or excluded.

Method: Participated in this study 30 individuals with psychoactive substances use, aged between 24 and 65 years $(M \pm D P=43,03 \pm 10,10)$, most of them male $(80,0 \%)$, with no source of income (46.7\%) or supported by social policy measures (53.3\%). The main substance of use is heroin (63.3\%). We assessed the structural, functional and contextual-relational dimensions of their networks with the Social Personal Networks Analysis Tool.

Results: The social networks of the participants are composed by 7 elements, in average, predominating the family members; they are fragmented, consisting essentially by members of the primary networks, although, the secondary networks represent about $1 / 4$ of the members. Reciprocity and the level of perceived social support are high, such as emotional and informational support. Most individuals report having members in the social network with drug abuse $(77,0 \%)$, relationships that occupy more than $20,0 \%$ of the network size. We analyzed comparatively the networks according to material and shelter deprivation, and no significant differences emerged ( $p>0,05$ ), although the networks' profiles suggest different strategies of support.

Conclusions: The study confirms the relevance of social networks assessment in the population with addictive behaviors and dependencies. Social intervention planning should integrate the network characteristics that are relevant to support the reduction of substance abuse and associated risks, as well as the promotion of social well-being and citizenship. 


\section{INTRODUÇÃO}

Vivemos num emaranhado relacional. Como os pontos se ligam entre si, através de linhas, as pessoas serão os pontos e as linhas serão as interações que se vão tecendo ao longo da vida. As redes relacionais formam a trama da sociedade e das sociabilidades, configurando-se enquanto sistemas sociais de suporte e de reconhecimento identitário.

Segundo a perspetiva ecológica do desenvolvimento humano, a rede social do indivíduo integra um conjunto de sistemas sociais que o influenciam e que o próprio influencia permanentemente. Esta perspetiva permite-nos ver para além das causas tidas como mais imediatas das situações e comportamentos das pessoas, já que tem em conta as complexas influências históricas e ambientais que aí intervêm, tanto direta como indiretamente (Lacroix, 1990). Numa perspetiva egocentrada, Sluzki (1996, p. 42) define rede social pessoal como o conjunto de "todas as relações que um indivíduo percebe como significativas ou que define como diferenciadas da massa anónima da sociedade. Essa rede corresponde ao nicho interpessoal da pessoa e contribui substancialmente para seu próprio reconhecimento como indivíduo e para a sua autoimagem". As redes sociais são consideradas "sistemas abertos que, através de um intercâmbio dinâmico entre os seus membros e os elementos de outros grupos sociais, potencializam outros recursos" (Alarcão e Sousa, 2007, p. 356). Assim, tais sistemas proporcionam socialização.

Quando nos reportamos à magnitude dos problemas ligados aos comportamentos aditivos e dependências ( $C A D$ ), para além do evidente problema de saúde, este tem implicações holísticas, encontrando-se associado a problemas de ordem emocional, comportamental, relacional e social. Em termos sociais, referimo-nos a um conjunto de processos e de retrocessos na socialização e na capacitação para a autonomia pessoal e participação plena na sociedade. Nestes processos e retrocessos, Ló (2011) refere que no percurso da dependência assiste-se à degradação dos laços familiares e de amizade, com a escola, a empresa e outras instituições numa progressiva substituição dos quadros de valores de referência. Verifica-se, em grande parte dos casos, um afastamento do toxicodependente do exercício dos direitos e deveres de cidadania, e a sua integração num sistema social cuja estrutura se alicerça num padrão sociocultural específico, organizado exclusivamente em torno da tríade procura-ofertaconsumo de drogas.

A exclusão social associada aos CAD é um fenómeno que confirma, geralmente, uma situação deficitária a nível de recursos (económicos, sociais, culturais) que dificultam a participação social do indivíduo. É reprodutora de si mesma, pois a cisão social proporciona, por sua vez, uma maior marginalização, não só materialmente mas também simbolicamente, conduzindo a um sentimento de autoexclusão (Andrade et al., 2007). Independentemente do consumo de drogas ser uma das causas ou consequências do processo de exclusão social, os CAD são mais um fator potenciador da manutenção dessa realidade.

O processo de exclusão "empurra" os sujeitos para as ditas margens da sociedade, isto é, dos modos de vida que se desejariam habituais e socialmente englobantes, margens estas excluídas da participação social e da cidadania, forjando paradoxalmente o que Leandro e Ferreira (2011) designam por laço social e cultura de urgência. A inclusão faz-se através da aproximação e socialização em grupos excluídos, será, portanto, a "inclusão na exclusão".

Este processo de desintegração e de desqualificação faz-se muitas vezes associado a ruturas nos sistemas sociais básicos e nos laços sociais, configurando uma forma de dessocialização (Castel, 2003; Costa, 2004; Leandro e Ferreira, 2011; Paugam, 2000). López (2010) entende que as pessoas com CAD são especialmente suscetíveis de mostrar fragilidades na sua rede social, verificando-se frequentemente situações de vulnerabilidade relacional associadas à ausência ou debilidade dos vínculos de inserção comunitária. Durante um longo período estas pessoas tiveram redes com baixos níveis de integração e grande estigma social, o que gerou uma fratura relacional com a sua rede familiar e de amizade.

A expressão da vulnerabilidade das redes sociais de pessoas com CAD é apresentada pelo seu tamanho pequeno, elevada densidade e conexões débeis (López, 2010), sendo essencialmente constituídas por relações familiares e institucionais (Panebianco, Gallupe, Carrington, Colozzi, 2016), vínculos sobrecarregados e com suporte enfraquecido (Cavalcante et al., 2012).

López (2010) refere ainda que é comum encontrar redes pessoais com vínculos fortes, normalmente nas relações familiares, apesar de muito saturadas, em que o indivíduo não consegue satisfazer as suas necessidades emocionais, materiais e de informação nos recursos do seu grupo primário. No que se refere às suas relações de amizade, a sua maioria apresenta igualmente o mesmo perfil de CAD.

Como a investigação na área da rede social pessoal de indivíduos com CAD é escassa, pretende-se, com este estudo, caracterizar a rede social pessoal de pessoas com CAD em situação de exclusão social, refletir sobre a qualidade dos vínculos e possíveis formas de ativar esta rede. 


\section{MÉTODO}

\section{Procedimentos e amostragem}

Este é um estudo transversal que utiliza uma metodologia quantitativa do tipo descritiva e inferencial.

Foi solicitada a autorização para a utilização e adaptação às finalidades da investigação do Instrumento de Análise da Rede Social Pessoal (IARSP) a uma das autoras do instrumento. Solicitou-se também a autorização formal à instituição que permitiu a recolha de dados junto dos participantes, no âmbito de uma "equipa de rua" de um projeto de intervenção social. Foram apresentados os objetivos do estudo aos participantes e solicitada a sua colaboração, após consentimento informado e garantida a confidencialidade na recolha dos dados.

Os participantes foram selecionados com base no critério da acessibilidade, constituindo uma amostra não probabilística, por conveniência. A amostra integra indivíduos com comportamentos aditivos e dependências sinalizados pela instituição. A recolha de dados verificouse no período de fevereiro a abril de 2016, em Aveiro (Portugal). Os instrumentos foram administrados por uma das investigadoras, membro da equipa da instituição, em dois contextos de contacto habitual com esta população, de acordo com a disponibilidade dos inquiridos: no gabinete da equipa e na rua.

A análise estatística dos dados recolhidos na inquirição foi efetuada através do Statistic Package for the Social Sciences (IBM - SPSS), versão 23 para o Windows.

\section{Participantes}

Participaram no presente estudo 30 pessoas com comportamentos aditivos e dependências em risco de exclusão social, com apoio institucional, na sua maioria pessoas do sexo masculino $(n=24 ; 80,0 \%$ ), com idades compreendidas entre os 24 e os 65 anos $(M \pm D P=43,03 \pm$ $10,10)$, a maior parte na faixa etária de $35-44$ anos $(n=12$; 40,0\%). Quanto ao estado civil, 63,3\% $(n=19)$ são solteiros, $23,3 \%(n=7)$ divorciados e $13,4 \%(n=4)$ são casados e/ou em união de facto. Os participantes são todos de nacionalidade portuguesa, sendo 70,0\% $(n=21)$, residentes no distrito de Aveiro.

Quanto à escolaridade, $73,3 \%(n=22)$ possuem o ensino básico incompleto, $23,3 \%(n=7)$ o ensino básico completo e $3,3 \%(n=1)$ o ensino superior. A maior parte dos inquiridos refere não ter fontes de rendimento $(n=14$; $46,7 \%), 36,7 \%(n=11)$ têm direito ao rendimento social de inserção, $10,0 \%(n=3)$ têm o subsídio de desemprego e $6,6 \%(n=2)$ beneficiam de outras medidas de apoio social; na amostra, $36,7 \%(n=11)$ refere recorrer à atividade de "arrumar carros", regular ou pontualmente, como fonte suplementar de rendimento.
Quanto ao alojamento, $23,3 \%(n=7)$ residem num quarto alugado, $20,0 \%(n=6)$ em casa de familiares, $13,3 \%$ $(n=4)$ em casas abandonadas, com frequências inferiores registamos quem vive em casa alugada $(n=3 ; 10,0 \%)$, em casa cedida ( $n=3 ; 10,0 \%)$, na rua $(n=3 ; 10,0 \%)$, em habitação social ( $n=2 ; 6,6 \%)$, em casa própria $(n=1 ; 3,3 \%)$ e em pensão ( $n=1 ; 3,3 \%)$. Sete dos inquiridos $(23,3 \%)$ encontram-se numa situação de sem-abrigo. A maioria $(n=$ $17 ; 56,7 \%)$ vive só; de entre os que vivem em coabitação, $26,7 \%(n=8)$ vivem com o companheiro/a, seguido de $6,7 \%$ $(n=2)$ com um elemento da família nuclear (mãe e/ou pai), $6,7 \%(n=2)$ com a companheira e outros elementos da família nuclear e 3,3\% ( $n=1)$ com um tio.

Metade da amostra $(n=15 ; 50,0 \%)$ referiu ter problemas judiciais pendentes (como liberdade condicional e trabalho comunitário) e 73,3\% ( $n=22)$ referem ter problemas de saúde (como doenças mentais e doenças infetocontagiosas diagnosticadas).

\section{Instrumentos}

O estudo usou como instrumentos de recolha de dados um breve inquérito para caracterização dos participantes (dados gerais e perfil de consumo) e o Instrumento de Análise da Rede Social Pessoal (IARSP) (Guadalupe, 2009) para caracterizar as dimensões estrutural, funcional e relacional-contextual das suas redes.

O IARSP foi adaptado para o estudo, tendo usado o seguinte gerador de rede: "refira o nome das pessoas com que se relaciona, são significativas na sua vida e o/a apoiam", seguindo-se a questão sobre o vínculo relacional estabelecido entre o sujeito e cada uma das pessoas enunciadas como membros na sua rede, com os seguintes campos relacionais: família (com caracterização do vínculo), amigo/a, vizinho/a, relação de trabalho ou de estudo, técnico/a (relação institucional).

Na dimensão estrutural caracterizámos o tamanho da rede; a composição da rede (através do número de campos relacionais, do tamanho e da proporção dos campos relacionais no tamanho da rede); e a densidade da rede (que expressa percentualmente a interconexão entre os membros da rede, podendo variar entre zero e cem, categorizando como redes dispersas aquelas que apresentam valores inferiores a $33,33 \%$, fragmentadas entre $33,33 \%$ e $79,99 \%$ e coesas de $80,0 \%$ a $100,0 \%$ ); e as mudanças percebidas no tamanho da rede nos últimos dois anos (5. "o número de pessoas com que relaciona é muito maior do que antes", 4. “...é pouco maior...", 3. “...é aproximadamente o mesmo", 2. “...é pouco menor ..." e 1. “...é muito menor...").

Na dimensão funcional avaliámos o nível de apoio percebido com uma escala de Likert de frequência de apoio com 5 pontos (1."nunca", 2. "raramente", 3. “algumas vezes", 4. “frequentemente" e 5. "sempre”) 
quanto ao apoio emocional, material e instrumental e informativo; a reciprocidade de apoio avaliada através de 5 níveis (5. "dá apoio à maior parte destas pessoas", 4. “...a algumas...", 3. “...a poucas...", 2. “...a muito poucas..." e 1. "não dá apoio a nenhuma...").

Quanto às características relacionais-contextuais avaliámos a frequência de contactos (5. "diariamente", 4. "algumas vezes por semana", 3. "semanalmente", 2. "algumas vezes por mês" e 1. "algumas vezes por ano"); a frequência de contactos por campo relacional; a dispersão geográfica (5. "na mesma casa", 4. "no mesmo bairro/rua", 3. "na mesma terra", 2. "até 50 Km" e 1. "a mais de $50 \mathrm{Km}$ "); a dispersão geográfica por campo relacional; e a homo/heterogeneidade de elementos com CAD na rede, onde se questiona se o elemento da rede apresenta ou apresentou consumos de substâncias psicoativas (1. "sim" e 2. "não") e a proporção na rede social pessoal.

\section{RESULTADOS}

\section{Perfil de consumo}

Dos participantes neste estudo, 19 referem a heroína $(63,3 \%)$ como substância principal de consumo, 4 a cocaína (13,3\%), 4 o álcool (13,3\%), 2 a cannabis $(6,7 \%)$ e 1 as benzodiazepinas (3,3\%). A maioria dos inquiridos $(n=26$; $86,7 \%$ ) apresenta o que é designado por policonsumo consumo de mais do que uma droga ou tipo de droga por um mesmo indivíduo - consumo simultâneo ou sequencial (EMCDDA, 2002), 83,3\% ( $n=25)$ referem que consomem regularmente substâncias psicoativas e 16,7\% ( $n=5)$ esporadicamente. A via de consumo é maioritariamente fumada ( $n=18 ; 60,0 \%)$, seguida da injetada $(n=7 ; 23,3 \%)$ e oral $(n=5 ; 16,7 \%)$ (Tabela 1$)$. A média do tempo de consumo é de 21 anos $(M \pm D P=21,33 \pm 9,27)$. A maioria dos inquiridos está ou já esteve numa unidade de tratamento ( $n=23 ; 76,7 \%$ ), como Equipas de Tratamento, Unidades de Desabituação ou Comunidades Terapêuticas.

Tabela 1

Características do consumo

\begin{tabular}{llcc}
\hline $\mathbf{n = 3 0}$ & & $\mathbf{n}$ & $\%$ \\
\hline \multirow{3}{*}{ Substância principal } & Heroína & 19 & 63,3 \\
de consumo & Cocaína & 4 & 13,3 \\
& Álcool & 4 & 13,3 \\
& Cannabis & 2 & 6,7 \\
& Benzodiazepinas & 1 & 3,3 \\
\hline \multirow{2}{*}{ Policonsumo } & Apresneta & 26 & 86,7 \\
& Não apresenta & 4 & 13,3 \\
\multirow{2}{*}{ Via de consumo } & Fumada & 18 & 60,0 \\
& Injetada & 7 & 23,3 \\
\hline Frequência de & Oral & 5 & 16,7 \\
consumo & Regular & 25 & 83,3 \\
\hline
\end{tabular}

\section{Características estruturais da rede social pessoal}

O tamanho da rede é um indicador importante quando se estudam os aspetos estruturais da rede de suporte social. Os inquiridos apresentam, em média, 7 elementos $(M \pm D P=7 \pm 2,73)$, variando entre 3 a 15 membros. Quanto à composição da rede, as redes são, em média, constituídas por dois campos relacionais distintos $(M=1,90)$,tendo mais membros o campo das relações familiares $(M=3,97)$, seguido do campo das relações de amizade e das relações institucionais (respetivamente, $M=$ 1,63 e $M=1,37$ ). O campo das relações de vizinhança apresenta um número de membros residual e o das relações de trabalho e/ou estudo é nulo. Relativamente à distribuição da rede, isto é, à proporção de membros por campo relacional no tamanho da rede, as relações familiares são as que têm um peso maior na rede $(M=$ $54,8 \%)$, seguidas das relações institucionais $(M=23,13 \%)$ e das relações de amizade $(M=20,2 \%)$ (Tabela 2$)$.

A densidade da rede permite-nos representar a conexão entre os membros da rede, acedendo qualitativamente à disposição da mesma relativamente ao sujeito central e dos membros entre si. A densidade média das redes dos inquiridos é de 70,93\% ( $D P=25,18)$. Análises adicionais indicam-nos que a maioria dos sujeitos apresenta redes fragmentadas ( $n=16 ; 53,3 \%$ ), isto é, que apresentam níveis de densidade entre os 33,3\% e os 79,9\% de interconexão.

Avaliámos ainda a mudança percebida no tamanho de rede nos últimos dois anos, tendo $26,7 \%(n=8)$ referido que o número de pessoas com que se relaciona é aproximadamente o mesmo, 23,3\% $(n=7)$ dos inquiridos indica que é muito menor do que antes, 20,0\% $(n=6)$ que é pouco menor que antes, $20,0 \%(n=6)$ que é pouco maior que antes, e 10,0\% $(n=3)$ que é muito maior que antes.

\section{Tabela 2}

Características estruturais da rede social pessoal

\begin{tabular}{lcccc}
\hline $\mathbf{n}=30$ & $M$ & $D P$ & Mín. & Máx. \\
\hline Tamanho da rede & 7,00 & 2,73 & 3 & 15 \\
Campos relacionais (n. ${ }^{\circ}$ ) & 1,90 & 0,76 & 1 & 3 \\
Composição da rede (a) & & & & \\
$\quad$ Relações familiares & 3,97 & 3,05 & 0 & 11 \\
Relações de amizade & 1,63 & 3,08 & 0 & 13 \\
Relações de trabalho (b) & 0 & 0 & 0 & 0 \\
Relações de vizinhança & 0,10 & 0,31 & 0 & 1 \\
Relações institucionais & 1,37 & 1,73 & 0 & 6 \\
Distribuição da rede (c) (em \%) & & & & \\
$\quad$ Relações familiares & 54,80 & 35,22 & 0 & 100 \\
Relações de amizade & 20,20 & 32,58 & 0 & 100 \\
Relações de trabalho (b) & 0 & 0 & 0 & 0 \\
Relações de vizinhança & 1,90 & 5,82 & 0 & 20,0 \\
Relações institucionais & 23,13 & 29,35 & 0 & 100 \\
Densidade da rede (em \%) & 70,93 & 25,18 & 19,05 & 100 \\
\hline
\end{tabular}

Notas: $M=$ Média; $D P$ = Desvio Padrão; Min = Mínimo; Máx = Máximo.

(a) n. ${ }^{\circ}$ de membros por campo relacional

(b) relações de trabalho e/ou de estudo

(c) proporção do campo relacional no tamanho 


\section{Características funcionais da rede social pessoal}

O apoio social percebido na rede foi avaliado com base numa escala de cinco pontos, que reflete o nível no qual o sujeito refere receber apoio emocional, material e/ou instrumental e informativo dos membros da sua rede. Os resultados indicam que os inquiridos percebem um nível mais elevado no apoio emocional $(M \pm D P=4,20 \pm$ 1,10), seguido do apoio informativo $(M \pm D P=3,97 \pm 1,22) \mathrm{e}$ do apoio material e instrumental $(M \pm D P=3,40 \pm 1,28)$.

A reciprocidade do apoio é elevada $(M \pm D P=4,17 \pm$ 0,99; $\operatorname{Min}=1 ; \operatorname{Max}=5$ ), dado que $43,3 \%$ dos inquiridos refere que dá apoio à maior parte dos elementos da sua rede, $40,0 \%$ a algumas pessoas, $10,0 \%$ a poucas destas pessoas, 3,3\% referem que dão apoio a muito poucas pessoas e 3,3\% que não dão apoio aos elementos da sua rede (Tabela 3 ).

Tabela 3

Características Funcionais da Rede Social Pessoal

\begin{tabular}{lcc}
\hline $\mathbf{n}=\mathbf{3 0}$ & $\mathbf{M}$ & $\mathbf{D P}$ \\
\hline Nível de apoio & 4,20 & 1,10 \\
Apoio emocional & 3,40 & 1,28 \\
Apoio material e instrumental & 3,97 & 1,22 \\
Apoio informativo & 4,17 & 0,99 \\
\hline Reciprocidade de apoio & $\mathbf{n}$ & $\%$ \\
\hline Reciprocidade de apoio & 13 & 43,3 \\
\hline Dá apoio à maior parte destas pessoas & 12 & 40,0 \\
Dá apoio a algumas destas pessoas & 3 & 10,0 \\
Dá apoio a poucas destas pessoas & 1 & 3,3 \\
Dá apoio a muito poucas destas pessoas & 1 & 3,3 \\
Não dá apoio a nenhuma destas pessoas & &
\end{tabular}

Notas: $M=$ Média; $D P=$ Desvio Padrão

\section{Características relacionais-contextuais da rede social pessoal}

$\mathrm{Na}$ frequência de contactos dos inquiridos com os membros da sua rede, $43,3 \%(n=13)$ refere que contactam algumas vezes por semana, $26,7 \%(n=8)$ estabelecem contactos semanalmente e $13,3 \%(n=4)$ algumas vezes por mês. Quanto à média da frequência de contactos por campo relacional, destacamos as relações de vizinhança com contactos diários $(M \pm D P=4,67 \pm 0,58)$, as relações de amizade $(M \pm D P=4,42 \pm 1,00)$ e as relações institucionais $(M \pm D P=3,71 \pm 1,07)$ com contactos algumas vezes por semana.

Quanto à dispersão geográfica da rede, $53,3 \%(n=16)$ dos elementos das redes dos sujeitos residem em Aveiro. Na distância geográfica por campo relacional destacamos as relações de vizinhança $(M \pm D P=4 \pm 0,00)$ e as relações de amizade $(M \pm D P=3,15 \pm 0,90)$ como as geograficamente mais próximas do sujeito, as relações institucionais $(M \pm D P=3 \pm 0,00)$ e as relações familiares $(M \pm D P=2,52 \pm 1,28)$ situam-se em média na cidade de Aveiro.

Quanto à homo/heterogeneidade de elementos com CAD na rede dos inquiridos, $23(77,0 \%)$ referiram que têm elementos com CAD $(M \pm D P=1,43 \pm 1,43$, de 0 a 6 membros), a proporção destes elementos corresponde a uma média de $22,0 \%$ no tamanho total da rede dos inquiridos (Tabela 4).

\section{Tabela 4}

Características Relacionais-Contextuais da Rede Social Pessoal

\begin{tabular}{|c|c|c|c|c|}
\hline$n=30$ & $n$ & $\%$ & $M$ & DP \\
\hline Frequência de contactos & & & 3,37 & 1,07 \\
\hline Diariamente & 3 & 10,0 & & \\
\hline Algumas vezes por semana & 13 & 43,3 & & \\
\hline Semanalmente & 8 & 26,7 & & \\
\hline Algumas vezes por mês & 4 & 13,3 & & \\
\hline Algumas vezes por ano & 2 & 6,7 & & \\
\hline \multicolumn{5}{|l|}{ Frequência de contactos } \\
\hline por campo relacional & & & 2,78 & 1,34 \\
\hline Relações familiares & & & 4,42 & 1,00 \\
\hline Relações de amizade & & & - & - \\
\hline Relações de trabalho/estudo & & & 4,67 & 0,58 \\
\hline Relações de vizinhança & & & 3,71 & 1,07 \\
\hline \multicolumn{5}{|l|}{ Relações institucionais } \\
\hline Dispersão geográfica na rede & & & 2,77 & 0,86 \\
\hline Na mesma casa & - & - & & \\
\hline No mesmo bairro/rua & 5 & 16,7 & & \\
\hline Na mesma terra & 16 & 53,3 & & \\
\hline Até $50 \mathrm{Km}$ & 6 & 20,0 & & \\
\hline A mais de $50 \mathrm{Km}$ & 3 & 10,0 & & \\
\hline $\begin{array}{l}\text { Dispersão geográfica por } \\
\text { campo relacional }\end{array}$ & \multicolumn{3}{|c|}{ campo relacional } & \\
\hline Relações familiares & & & 2,52 & 1,28 \\
\hline Relações de amizade & & & 3,15 & 0,90 \\
\hline Relações de trabalho/estudo & & & - & - \\
\hline Relações de vizinhança & & & 4,00 & 0,00 \\
\hline Relações institucionais & & & 3,00 & 0,00 \\
\hline \multicolumn{5}{|l|}{$\begin{array}{l}\text { Homo/heterogeneidade de } \\
\text { elementos com CAD na rede }\end{array}$} \\
\hline Elementos com CAD & & & 1,43 & 1,43 \\
\hline Proporção de elementos com CAD & & & $22,43 \%$ & $25,06 \%$ \\
\hline
\end{tabular}

Notas: $M=$ Média; $D P=$ Desvio Padrão

\section{Características da rede social pessoal segundo a privação material e a situação de abrigo}

Tendo em conta as características dos sujeitos no que concerne à situação de vulnerabilidade e de exclusão social, fomos analisar comparativamente as médias relativas às características das redes no que concerne à privação material (Tabela 5) e de abrigo (Tabela 6). No entanto, não foram encontradas diferenças significativas entre as médias nas 
características das redes nos grupos em comparação, com uma única exceção relativa à maior proporção de elementos com CAD entre os que dispõem de fontes de rendimento $(p<0,001)$. Apesar das diferenças não serem estatisticamente significativas, provavelmente atendendo à elevada variância na maior parte das variáveis, a análise das redes segundos estas duas características, afigura-se como relevante para reflexão, atendendo a que podem corresponder a distintos perfis de ativação dos recursos da rede, registando-se tendências distintas na observação descritiva das médias.
Entre os indivíduos que apresentam rendimentos, as suas fontes são medidas de política social, na sua totalidade. Os sujeitos sem rendimentos apresentam redes maiores $(M=$ $7,71$ vs. $M=6,38)$, mais diversas nos campos relacionais ( $M$ $=2,14$ vs. $M=1,69)$, centradas nas relações familiares $(M=$ $63,14 \%$ vs. $M=47,50 \%)$ e institucionais $(M=27,14$ vs. $M=$ 19,63), percebendo maiores níveis de apoio. Os sujeitos com rendimentos apresentam maior proporção de relações amicais $(M=31,63$ vs. $M=7,14)$ e de membros que partilham CAD ( $M=29,75$ vs. $M=14,07)$ nas suas redes, assim como uma perceção de maior nível de reciprocidade $(M=4,31$ vs. $M=4,00)$.

\section{Tabela 5}

Características da Rede Social Pessoal Segundo a (In)existência de Fontes de Rendimento

\begin{tabular}{|c|c|c|c|c|c|}
\hline & \multicolumn{2}{|c|}{$\begin{array}{l}\text { Com fonte de rendimentos } \\
\qquad n=16(53,3 \%)\end{array}$} & \multicolumn{2}{|c|}{$\begin{array}{l}\text { Sem fonte de rendimentos } \\
\qquad n=14(46,7 \%)\end{array}$} & \multirow[b]{2}{*}{$U$} \\
\hline & $M$ & DP & $M$ & DP & \\
\hline \multicolumn{6}{|l|}{ Características Estruturais } \\
\hline Tamanho da rede & 6,38 & 3,12 & 7,71 & 2,09 & $70,500^{\text {n.s. }}$ \\
\hline Densidade & 69,30 & 29,07 & 72,79 & 20,79 & $106,500^{\text {n.s. }}$ \\
\hline Campos relacionais & 1,69 & 0,60 & 2,14 & 0,86 & $77,000^{\text {n.s. }}$ \\
\hline \multicolumn{6}{|c|}{ Distribuição da rede (proporção - \%) } \\
\hline Relações familiares & 47,50 & 37,78 & 63,14 & 31,31 & $82,000^{\text {n.s. }}$ \\
\hline Relações de amizade & 31,63 & 40,39 & 7,14 & 11,91 & $81,000^{\text {n.s. }}$ \\
\hline Relações de vizinhança & 1,25 & 5 & 2,64 & 6,74 & $103,500^{\text {n.s. }}$ \\
\hline Relações de trabalho & 0 & 0 & 0 & 0 & $112,000^{\text {n.s. }}$ \\
\hline Relações institucionais & 19,63 & 32,73 & 27,14 & 25,55 & $87,000^{\text {n.s. }}$ \\
\hline \multicolumn{6}{|l|}{ Características Funcionais } \\
\hline Apoio emocional & 4,13 & 1,15 & 4,29 & 1,07 & $106,000^{\text {n.s. }}$ \\
\hline Apoio material e instrumental & 3,31 & 1,25 & 3,50 & 1,35 & $101,500^{\text {n.s. }}$ \\
\hline Apoio informativo & 3,69 & 1,35 & 4,29 & 0,99 & $85,500^{\text {n.s. }}$ \\
\hline Reciprocidade de apoio & 4,31 & 0,87 & 4,00 & 1,11 & $93,000^{\text {n.s. }}$ \\
\hline \multicolumn{6}{|c|}{ Características Relacionais-Contextuais } \\
\hline Frequência de contactos & 3,50 & 1,10 & 3,21 & 1,05 & $97,500^{\text {n.s. }}$ \\
\hline Distância da residência & 2,75 & 0,78 & 2,79 & 0,98 & $105,500^{\text {n.s. }}$ \\
\hline Proporção CAD & 29,75 & 32,60 & 14,07 & 5,78 & $96,000^{\text {n.s. }}$ \\
\hline
\end{tabular}

Notas: As fontes de rendimentos são medidas de política social. $U$ = Teste de Mann-Whitney; n.s. = $p$ não significativo; * $p=0,001$

Os indivíduos que se encontram sem-abrigo apresentam características morfológicas das redes muito semelhantes com as dos que têm abrigo, apenas divergindo no que toca às relações de vizinhança que não são apresentadas pelos primeiros. Os sujeitos com CAD sem abrigo apresentam uma rede maior $(M=8,14$ vs. $M=6,65)$ e com composição idêntica às dos que têm abrigo, reconhecem na rede níveis disponíveis de apoio instrumental e informativo mais elevados (respetivamente: $M=3,86$ vs. $M=3,26 ; M=4,43$ vs. $M$ = 3,83), mas menor apoio emocional e menor reciprocidade (respetivamente: $M=3,86$ vs. $M=4,30$; $M=3,71$ vs. $M=4,30$ ), percebidos como mais baixo do que pelos outros. Os que têm alojamento apresentam nas suas redes um percentual maior de membros com CAD $(M=26,74$ vs. $M=8,29)$. 
Tabela 6

Características da Rede Social Pessoal Segundo a Situação de Abrigo

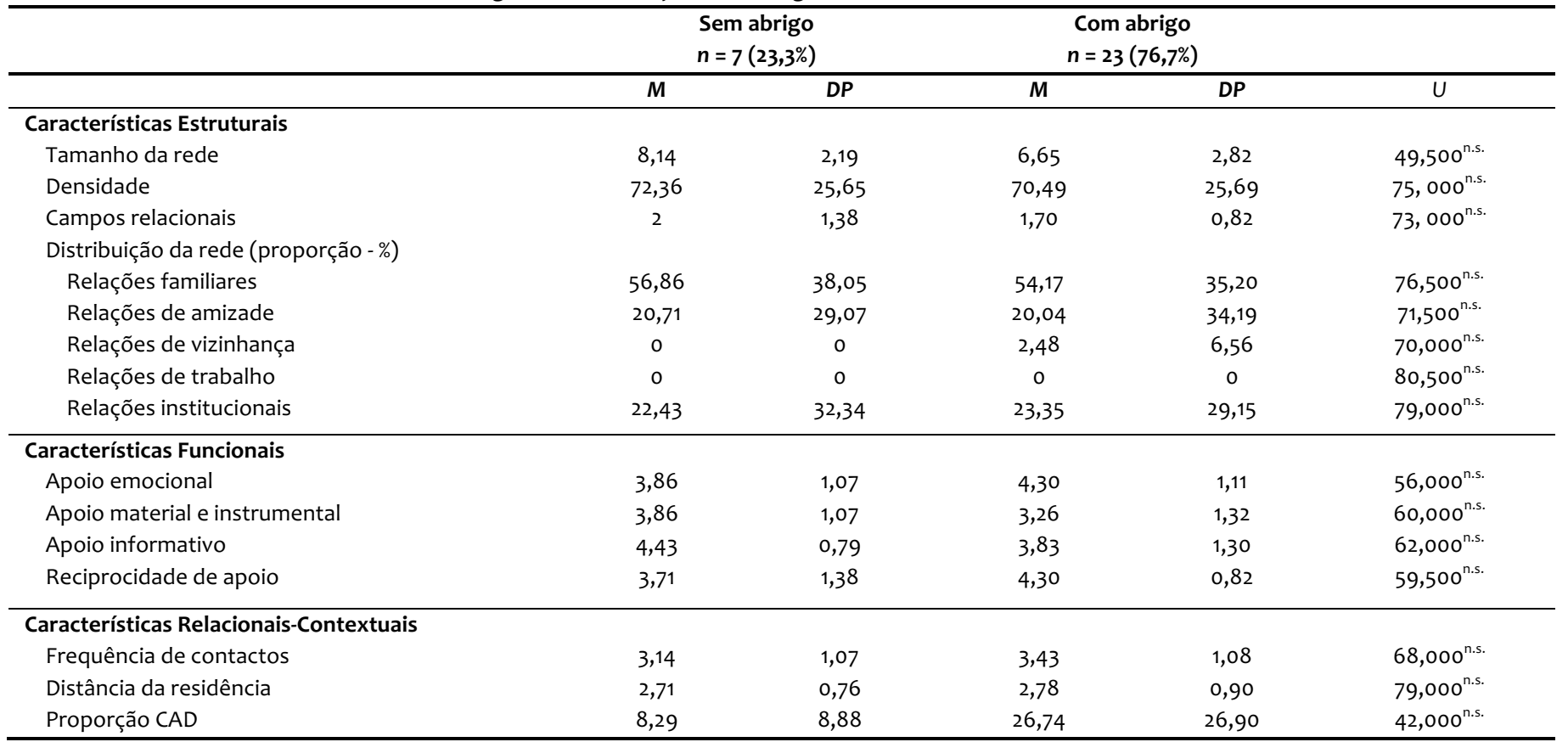

Notas: $U$ = Teste de Mann-Whitney; n.s. = $p$ não significativo; * $p=0,001$

\section{DISCUSSÃO}

Os resultados deste estudo permitem uma aproximação às principais características das redes sociais de pessoas com CAD em risco de exclusão social.

Segundo estimativas para o contexto nacional (SICAD, 2013), cerca de 7,1\% dos habitantes de 15-64 anos apresentavam consumo problemático/de alto risco de drogas em 2012 (sendo estas opiáceos, cocaína e/ou anfetaminas/metanfetaminas) e 2,2\% da população jovem adulta residente em Portugal apresentava sintomas de dependência de consumos endovenosos. Segundo o Diagnóstico "Álcool e Drogas" do concelho de Aveiro (CRIA, 2012), foram identificados 170 indivíduos em risco de exclusão, sendo estes caracterizados como arrumadores de carros, sem-abrigo e/ou trabalhadores do sexo com $C A D$, com nível socioeconómico e escolaridade baixa, problemas habitacionais, facilidade de acesso a substâncias e com famílias desestruturadas ou sem enquadramento sociofamiliar. A nossa amostra representa, assim, $18,0 \%$ dos casos sinalizados neste concelho.

Neste estudo, 36,7\% dos indivíduos inquiridos recorrem à atividade de "arrumar carros" como forma de obtenção de rendimento, sendo que este tipo de atividade representa uma ocupação com horários e locais fixos, uma rotina diária. Matias e Fernandes (2009) realizaram um estudo em que a análise dos dados permitiu perceber como estes sujeitos entendem a forma como são vistos pela comunidade, referindo que "são socialmente vistos como criminosos, quer na sua qualidade de toxicodependente quer na qualidade de toxicodependente arrumador" e apontados como "figura de ameaça" (Matias e Fernandes, 2009, p.15). No entanto, ser arrumador tanto pode ser visto como a desistência do indivíduo como a busca de dignidade ao insistir em permanecer no circuito económico da cidade, assumindo uma atividade da chamada "economia paralela", em vez de se esconder ou se auto excluir.

Outra característica mencionada no diagnóstico do CRIA (2012) prende-se com a questão da população semabrigo; $23,3 \%$ dos participantes com CAD encontram-se numa situação de sem-abrigo. Rosa e Guadalupe (2015, p. 187), sobre esta questão, referem que "a situação dos sem-abrigo conjuga privação, destituição, exclusão e dessocialização. Porque resultante da confluência múltipla de ruturas e desvinculações, que se mantêm no tempo, às quais se associam redes frágeis e reduzidas à dimensão instrumental, não concorre de forma positiva com a manutenção de laços sociais". A complexidade destas situações reclama o desenvolvimento de estratégias de intervenção capazes de se mobilizar as redes sociais (formais e informais) no sentido da (re)construção dos laços e cidadania.

Da análise comparativa efetuada entre os sujeitos em situação de sem abrigo e com abrigo e entre os indivíduos 
com e sem fonte de rendimentos, não ressaltaram diferenças estatisticamente significativas, mas chamaramnos a atenção algumas tendências de configuração e de recursos percebidos nas redes que nos parecem necessitar de um maior aprofundamento em futuros estudos. Isto porque entendemos que a privação material e habitacional, que colocam em causa direitos constitucionais e a dignidade da pessoa humana, podem equivaler a distintos perfis de necessidades e correspondente procura e ativação dos recursos da rede.

Teoricamente sabemos que uma rede de suporte sem recursos pode contribuir para um reforço da vulnerabilidade social e que os contextos de vulnerabilidade são marcados pela capacidade muito limitada de partilha de recursos nas redes informais. Os laços sociais precários e a diminuição de recursos sociais têm tendência a associar-se (Born e Lionti, 1996), havendo uma forte componente de reprodução social quando o suporte é eminentemente entregue a fontes informais, isto é, à rede primária, também afetada pelos fatores de vulnerabilidade que reconhecemos nos sujeitos acompanhados institucionalmente e que participaram no presente estudo. As trajetórias de exclusão dos indivíduos fazem-se no seio de uma rede, também ela (isto é, os seus membros) excluída frequentemente de alguns dos domínios considerados básicos para a inclusão social e cidadania. A população em estudo é caracterizada por longas histórias de consumo e de tratamento, com policonsumo e um consumo regular de substâncias psicoativas. Num estudo de Fernandes, Pinto e Oliveira (2006) fizeram um retrato dos utilizadores de drogas de rua, apoiados por equipas de rua e centros de acolhimento, tendo verificado que estes indivíduos tinham histórias de consumo com mais de 10 anos, várias tentativas frustradas de tratamento, rutura familiar, desligamento da rede de cuidados e ligação ao sistema judicial. Godinho (2007) num estudo sobre população semabrigo toxicodependente, que vivia temporariamente em centro de acolhimento, concluiu que a idade média de consumo da amostra foi de 16 anos, enquanto nos utentes com CAD acompanhados no Centro de Atendimento Toxicodependentes (CAT) era de 15 anos. Andrade et al. (2007), num estudo em que caracterizaram a população acompanhada por 15 equipas de rua, num total de 331 utentes, as características sociodemográficas referem que são na maioria do género masculino, solteiros, nacionalidade portuguesa, com habilitações literárias ao nível do ensino básico, desempregados, como se observa com os participantes neste estudo. Estes autores ainda referem que relativamente à situação judicial, 36,5\% já tinham sido detidos pelo menos uma vez e $28,1 \%$ já tinham estado efetivamente presos, enquanto 39,4\% tinham situações para resolver com a justiça, que vai ao encontro da elevada percentagem $(50,0 \%)$ de problemas judiciais encontrada no nosso estudo.

As características da população encontradas pelos estudos anteriormente referidos refletem-se nas dos nossos participantes, registando-se um tempo médio de consumo superior (21 anos), diversos tipos de tratamentos efetuados e problemas judiciais também entre a maior parte dos sujeitos da amostra do presente estudo, acrescidos de comorbilidades em problemas de saúde.

A rede pessoal dos participantes tem, em média, 7 elementos configurando redes pequenas (Alarcão e Sousa, 2007; Sluzki, 1996), quando comparadas com as da população geral. Os participantes do estudo têm consciência da restrição no tamanho das redes nesta população, ao contrário do observado no estudo de Souza e Kantorski (2009), em que os inquiridos mostram surpresa com o pequeno número de vínculos. Panebianco et al. (2016) caracterizaram a rede de 80 indivíduos com CAD que passaram por tratamentos, e concluíram que os abstinentes têm redes maiores em termos de laços com família e comunidade $(M=9)$, enquanto os que tiveram recaídas têm redes mais pequenas $(M=6)$, essencialmente constituídas por laços familiares e institucionais, o que também acontece na nossa amostra.

A distribuição da rede, segundo os campos relacionais, dá-nos a indicação da proporção ocupada pelos membros que compõem a rede tendo em conta a localização dos seus elementos. As relações familiares, as relações institucionais e as relações de amizade destacamse na amostra, tendo a família a proporção mais significativa, apresentando a maioria dos participantes 2 campos relacionais. Segundo Guadalupe (2009) as redes quando são muito localizadas num determinado campo relacional tendem a ser menos flexíveis e efetivas, pois apresentam menos opções do que aquelas que se estendem pelos vários campos relacionais, fazendo com que as pessoas se sintam muito dependentes entre si, sobrecarregando os seus elementos. Neste caso, a heterogeneidade é dominante na distribuição, emergindo dois campos da rede primária e um campo da rede secundária como dominantes. Evidencia-se ainda a residualidade da vizinhança e a inexistência das relações de trabalho e estudo, o que limita a expansão e a exploração de uma maior diversidade de recursos potenciais na rede.

As relações familiares assumem grande parte das relações percebidas, como sendo significativas e de suporte, seguidas das relações institucionais e das relações de amizade, enquanto as relações de vizinhança 
são pouco significativas e as de trabalho nulas. Estes dados parecem ir ao encontro do estudo de Andrade et al. (2007) que concluiram que o indivíduo com CAD avalia a relação com a sua a família como satisfatória (41,8\%), no entanto uma grande parte dos inquiridos tinha familiares que consumiam substâncias psicoativas (41,3\%), sendo estes sobretudo elementos da família direta do utente (83,6\%). Souza, Kantorski e Mielke (2006), num estudo com 6 indivíduos com CAD, também concluíram que a rede familiar era a única com a qual o indivíduo realmente contava, no entanto, por outro lado, as famílias descreviam-se como desgastadas.

Do mesmo modo que a família se vê afetada pela presença de um elemento com CAD, a dinâmica familiar é também equacionada como influenciando e favorecendo a manutenção deste comportamento (Botella, 2007). As interações com a família (de origem e atual) podem provocar uma dinâmica para o uso de substâncias, podendo agravar ou perpetuar o problema, assim como, por outro lado, ajudar a resolvê-lo. Neste sentido, uma cuidada avaliação das características familiares poderá ser um instrumento fundamental para planificar uma intervenção com as mesmas. São de salientar as condutas de sobreproteção e as dificuldades de promover a autonomização do indivíduo com CAD, as quais são necessárias para uma integração social satisfatória (Botella, 2007). Vanegas (2009) considera que a família é fundamental para a recuperação dos indivíduos com CAD, sendo que as intervenções devem ser orientadas para o restabelecimento das relações familiares. O autor defende que os riscos de CAD diminuem através de esquemas de suporte positivo, em se classifiquem as relações de autoridade, afeto e comunicação. No entanto, assinala os seguintes obstáculos: rede de apoio familiar inexistente, problemas de adição ou saúde mental em membros da família e dificuldades do meio envolvente em aceitar as fases da recuperação do indivíduo com CAD (Vanegas, 2009). Quando não é possível ativar a rede familiar devem procurar-se alternativas que garantam afeto, solidariedade e comunicação positiva no ambiente que rodeia os indivíduos em tratamento, como o apoio de amigos, vizinhos e instituições (Vanegas, 2009), explorando os recursos potenciais na rede extrafamiliar primária e secundária.

Cavalcante et al. (2012) mencionam ainda que os vínculos com a família trazem alguma ambiguidade, um relacionamento desgastado devido aos CAD, mas considerado fundamental para o processo de recuperação e sua manutenção. Após a rutura dos vínculos com os pares com $C A D$, pessoas que eram consideradas amigas, os entrevistados não conseguiram estabelecer vínculos de amizade consistentes, deixando clara a necessidade de intervenção com o intuito de aumentar a qualidade do suporte social, criando vínculos de amizade (Cavalcante et al., 2012). Os vizinhos aparecem no estudo de Cavalcante et al. (2012) como figuras negativas devido à descriminação e estigmatização que podem promover, o que pode eventualmente justificar o facto de no presente estudo os vizinhos serem residuais nas redes, para além da situação residencial errática

Souza et al. (2006) reportam que os participantes no seu estudo sentiram dificuldades em classificar alguns vínculos, visto que muitas vezes também são fontes de stress ou assumem características diferentes de acordo com a situação que vivem. Os amigos, no estudo de Souza et al. (2006), não emergiram com um papel central na rede social dos sujeitos, mesmo os pares com CAD não foram apontados como vínculos significativos, à semelhança do ocorrido no presente estudo. O rompimento dos vínculos com o trabalho surgiu como um fator causal ou como um processo de dependência às substâncias psicoativas. A principal rede operante nos vínculos destes indivíduos é sem dúvida as instituições, são conceituadas como uma parte da rede com a qual o sujeito obtém apoio, ajuda material, serviços e contactos sociais. Cavalcante et al. (2012) num estudo semelhante com 19 indivíduos com CAD referem ainda vínculos de apoio mais enfraquecidos, como a família, as amizades, o trabalho e os companheiros, vizinhos e ex-companheiros.

Segundo López (2010), estas pessoas estiveram inseridas em redes com baixo nível de integração e grande estigma social nas suas trajetórias, o que poderá ter gerado uma fratura relacional com a sua rede social familiar, de amizade e de trabalho. Segundo o autor, a situação de vulnerabilidade relacional caracteriza-se por: inserção do sujeito em redes vulneráveis com tamanho pequeno e elevada densidade, conexões débeis com outras redes; uma zona central da rede, normalmente ocupada pela família, que tende a estar saturada, na qual o sujeito procura satisfazer as suas necessidade materiais, emocionais e de informação; as relações de amizade são dominadas por sujeitos com CAD. Em muitas ocasiões as instituições favorecem uma certa endogamia, facilitando a criação de vínculos e consolidando situações de vulnerabilidade relacional. A este respeito Chadi (2000) menciona que as redes sociais institucionais podem ser definidas como organizações constituídas para cumprir com objetivos específicos, que satisfazem necessidades particulares e pontuais, que são canalizadas dentro de organismos criados especificamente para esses fins. Geralmente, quanto maior for a desconexão na rede primária, maior será a presença institucional na rede do 
indivíduo, pois a falta de uma rede primária estruturada empobrece o acesso aos recursos, sendo esta lacuna compensada pela rede secundária.

Quanto à interconexão entre os elementos da rede, os resultados apontam para níveis médios a elevados e redes fragmentadas, que segundo Guay (1984) são compostas por pequenos subgrupos relativamente independentes uns com os outros, muitas vezes situados num ou noutro campo da rede, sendo pouco frequentes os contactos entre os membros de diferentes subgrupos e rara a conexão entre os membros. A densidade da rede a nível qualitativo pode ser baixa, média ou alta. Sluzki (1996) refere ser o nível médio o que favorece a máxima efetividade do grupo, pois é aquele que permite a comparação entre as impressões e opiniões trocadas, sendo referidas como fomentando em maior medida o bem-estar dos indivíduos por apresentarem características que facilitam a adaptação à mudança (Guadalupe, 2009; Sluzki, 1996). No entanto, sendo estas redes compostas essencialmente por três campos relacionais, a fragmentação entre eles, pode eventualmente criar barreiras de interconexão que dificultem potenciais intervenções em rede. Relativamente ao apoio social percebido, os participantes referem um nível elevado de apoio ao nível emocional, que é caracterizado geralmente por trocas que envolvem atitudes emocionais positivas e um clima de compreensão, simpatia, empatia, estímulo e apoio, o que pressupõe a existência de relações de intimidade e proximidade; elevado apoio informativo, que se refere à partilha de informações pessoais ou sociais entre os elementos, assim como mostrar novas formas de pensar e agir perante a expectativa de ação futura; e um apoio material e instrumental moderado, conjunto de ações ou materiais que facilitam a realização das tarefas quotidianas (Sluzki, 1996). A reciprocidade do apoio percecionada é elevada, sendo que os participantes referem que dão apoio à maior parte dos elementos da sua rede. Os níveis funcionais elevados podem ser influenciados pela desejabilidade social e pela eventual necessidade de valorização do esforço dos membros da sua rede em compensar a sua situação de vulnerabilidade social e de doença.

Quando nos reportamos às propriedades específicas da dimensão relacional-contextual, dispersão geográfica da rede e frequência de contactos entre os elementos, percebemos que os participantes contactam algumas vezes por semana a maior parte dos elementos da rede e que estes residem na sua maior parte em Aveiro, sendo as relações de vizinhança e amizade as mais próximas geograficamente, portanto, esta conexão estabelecida nos vínculos na cidade Aveiro e na homogeneidade presente nas relações de amizade destes indivíduos leva à existência de similitudes patentes nos membros da rede em dimensões como atitudes, experiências e valores, o que vai ao encontro das ideias de Chadi (2000).

As funções de suporte favorecem a socialização do indivíduo, no entanto a integração pode aqui ser equacionada paradoxalmente, na medida em que a integração num contexto com CAD, num território excluído, pode constituir igualmente uma forma de exclusão social relativamente à comunidade em geral, como antes referimos. Quando analisamos a dispersão geográfica dos membros da rede por campo relacional, concluímos que a família a par com as instituições nem sempre são os campos relacionais que se encontram mais próximos dos participantes, o que nos leva a crer que, em eventual situação de crise, a distância a que se encontram as relações de amizade, pode ser significativo na procura de apoio imediato, e serão elementos fundamentais para a ativação da rede de suporte social.

Quanto à homogeneidade/heterogeneidade de elementos com CAD na rede dos participantes, 77,0\% referiram que têm elementos com CAD, representando, em média, $22,0 \%$ dos membros das suas redes, familiares ou amigos. Souza et al. (2011) realizaram um estudo onde se apurou a presença de elementos com CAD na família e outros grupos da rede social, tendo verificado que as principais necessidades deste grupo prendiam-se com o desenvolvimento de vínculos saudáveis, ampliação da rede social e estruturação do grupo, de modo a proporcionar benefícios em prol da efetiva reabilitação social. Os sujeitos em estudo contavam com uma rede social restrita, no geral com familiares, amigos ou examigos, também consumidores de drogas, sendo que todos já tinham sido submetidos a tratamentos anteriores, tinham histórico de recaídas e vínculos afetivos restritos. Para Souza et al. (2011) a presença de CAD na rede é considerada um fator de risco para recaída ou interrupção do tratamento. Os resultados apontam para o prejuízo na rede social dos indivíduos que tinham consumidores de drogas na rede, ou seja, a influência estava mais fortemente associada à continuidade do uso de drogas pelos indivíduos do que qualquer outro efeito benéfico, vindo da rede social dos participantes como, por exemplo, a frequência de contactos ou a disponibilidade de suporte. Outra característica referida no estudo aponta para a importância do cônjuge com CAD como factor potenciador da recaída.

A literatura sobre CAD sugere, geralmente, que a rede social tanto pode desencorajar como promover o abuso de drogas. No seu papel positivo, o suporte social está associado com compromisso e mudança social, 
tratamento e reinserção social. Mas a existência de uma rede social não é sinónimo da existência de suporte social (Guadalupe, 2009), havendo redes inócuas, não atuantes, e outras redes prejudiciais e até destrutivas. Sabemos que as relações interpessoais e a composição da rede estão associadas à iniciação em consumo de drogas, uso abusivos de substâncias psicoativas, recaídas e comportamentos de risco (El-Bassel, Chen e Cooper, 1998; Tucker et al., 2015). Tracy et al. (2016) acrescentam que o uso de substâncias na rede de amigos apresenta um risco elevado de pressão social e recaídas. É bastante provável que pessoas com CAD permaneçam nas suas redes após o tratamento, não sendo claro se são os indivíduos que são influenciados pelos membros com CAD da sua rede social ou se os seus próprios comportamentos aditivos e sociabilidade marginal influenciam a seleção de amigos.

Bohnert, Bradshaw e Latkin (2009) usaram a análise da rede social para estudar os CAD e confirmaram que o comportamento da rede social está associado ao uso de drogas do indivíduo, assim como os comportamentos de risco associados ao consumo. Davey et al. (2007) apuraram que os consumidores pela via endovenosa que têm outros consumidores via injetável na sua rede social, mais provavelmente vão continuar a usar drogas, quando comparados com indivíduos com poucos utilizadores de drogas na sua rede. Posto isto, estes autores consideram que o suporte social é o preditor mais forte de abstinência a seguir ao tratamento, ou seja, indivíduos que têm na sua rede membros que suportam a abstinência apresentam menos probabilidade de voltar a consumir após o tratamento; por outro lado, os indivíduos que interagem com outros consumidores depois do tratamento têm mais probabilidade de recair. Davey et al. (2007) também sugerem no seu estudo que quando existem muitos elementos ativos com CAD na rede podem existir consequências negativas, havendo menor probabilidade do indivíduo procurar tratamento, pois este tende a não sentir qualquer desejo ou motivação para a abstinência. Assim, a proporção elevada de membros com CAD nas redes dos participantes no presente estudo, é um factor central a ter em conta na planificação da intervenção.

A entrada para tratamento deve, assim, incluir uma intervenção ao nível da rede social, de forma a envolver o indivíduo no seu tratamento, assim como uma educação de pares e suporte necessário para enfrentar as potenciais recaídas (Davey et al., 2007; Garmendia, Alvarado, Montenegro e Pino, 2008; Silva, 2001). É possível que os indivíduos com CAD consigam perceber o problema em torno da sua rede, no entanto nem sempre são capazes de dar os passos necessários para solucionar esta situação. É necessário um apoio social mais constante, que acredite na diversificação dos recursos sociais e da comunidade como elementos-chave para gerar espaços de integração fomentando as redes sociais e o apoio mútuo.

Sendo que a adição a substâncias psicoativas representa para a sociedade atual um problema de saúde pública, para potenciar a otimização da intervenção nesta área é necessário uma maior compreensão da sua multicausalidade e uma intervenção mais integral (Vanegas, 2009). A rede social é tida unanimemente na literatura como um importante preditor da saúde e bemestar do indivíduo. Um estudo de Mericle (2014) sugere que indivíduos que estão ativamente envolvidos na sua comunidade e socialmente comprometidos com outros vivem mais tempo, são mais saudáveis, referindo que os fatores sociais, mais especificamente as características da rede social, estão associadas com a adoção de CAD, influenciando positivamente a abstinência, a entrada para tratamento e a sobriedade a longo prazo. A intervenção ao nível da rede social pessoal poderá, então, levar ao desenvolvimento de estratégias desenhadas para reduzir o consumo excessivo de substâncias psicoativas.

\section{CONCLUSÃO}

Os resultados deste estudo indicam que as redes sociais dos participantes, indivíduos com CAD em risco de exclusão, são pequenas, em média compostas por 7 pessoas, com dois campos relacionais (relações familiares, de amizade e/ou relações institucionais) e tendencialmente fragmentadas. O nível de apoio social percebido na rede e a reciprocidade são caracterizados como elevados, nomeadamente o apoio emocional e o informativo. Em média os sujeitos contactam comas relações de amizade e institucionais algumas vezes por semana, sendo os contactos com a família menos frequentes (algumas vezes por mês). Na distância geográfica, a maioria dos membros da rede dos participantes reside em Aveiro, no entanto os campos relacionais mais próximos são as relações de vizinhança e de amizade. As redes dos participantes apresentam uma proporção relevante de membros que partilham os CAD com o sujeito central.

Não podemos deixar de referir algumas limitações deste estudo, apesar de oferecer contributos nesta área de intervenção: seria importante aprofundar a análise das redes dos participantes, de forma a perceber os ganhos para o indivíduo com uma intervenção focada na sua rede social a longo prazo. No entanto, este estudo teve como pontos positivos o facto de levar os participantes a refletirem sobre a sua própria rede de suporte social, embora para alguns de forma dolorosa, possibilitou algum 
insight sobre os vários campos relacionais ao seu dispor, a qualidade e quantidade do suporte e a forma como se pode potenciar a sua rede social.

O fenómeno das drogas e o seu uso abusivo, como se verificou neste estudo, integra determinantes sociais e de saúde, constituindo um problema complexo com implicações no âmbito individual e na esfera pública. Esta complexidade requer um planeamento de intervenções de promoção de saúde com foco no empowerment do sujeito; para tal, as necessidades básicas dos indivíduos devem estar previamente asseguradas por políticas públicas que garantam alimentação, saúde, educação, lazer e trabalho (Souza et al., 2009). Portanto, a intervenção em rede deve assumir um carácter prioritário, sobretudo considerando os reflexos psicossociais que permeiam a questão das drogas no contexto afetivo, familiar e na comunidade, assegurando desta forma que os indivíduos tenham acesso aos cuidados básicos de saúde e a suporte social formal e informal.

Os obstáculos extrínsecos ao indivíduo existem, tais como a falta de preparação dos sistemas sociais para a compreensão deste fenómeno e das suas idiossincrasias, e o confronto sistemático com a manutenção do problema e a falta de resultados. Tais obstáculos constroem e reforçam as representações negativas sobre estes indivíduos e, consequentemente, a pouca participação e implicação das entidades nas respostas e soluções (Ló, 2011). No entanto, cabe aos profissionais que estão na chamada linha da frente e que assistem diariamente a mortes lentas e sofridas, terem a capacidade de criar empatia, assegurar dignidade no acesso aos serviços e direitos e potenciar os recursos que poderão gerar quadros de transformação e permitir percursos de vida sustentados. O papel dos interventores sociais é fundamental neste contexto, "a relação de confiança criada pelos técnicos das equipas de rua com os consumidores de drogas é absolutamente crucial para que o trabalho seja profícuo (...) para que esta relação seja construída a qualidade do perfil do técnico e as características da instituição são fundamentais, podendo a intervenção tornar-se contraditória e ineficaz" (Pinto e Peixoto, 2003, p.55). Barbosa (2011) acrescenta que as práticas do Serviço Social nesta área oscilam entre a regulação dos riscos psicoativos e o compromisso com a efetivação dos direitos da cidadania, através da relação com os utilizadores de drogas, pautada pela defesa da dignidade humana e proteção do direito à saúde. $O$ assistente social na sua ação apela à defesa da dignidade humana, reconhece o direito à diferença e promove a igualdade de oportunidade no acesso à saúde. Compromete-se a desenvolver uma relação de proximidade com os consumidores, uma função de mediação entre as necessidades expressas pelos consumidores e a burocracia dos recursos e práticas de empowerment, que valorizam a defesa dos direitos humanos e a promoção da cidadania ativa.

O papel do diagnóstico social micro e macrossocial destes problemas sociais complexos, o qual deve integrar a avaliação das redes de suporte social, tem um papel central no processo de intervenção.

Os resultados deste estudo sugerem que a caracterização da rede social pode ser uma "ferramenta" essencial na planificação da intervenção com pessoas com CAD. Considerando a importância do suporte e da interação social, uma possível intervenção para reduzir o uso abusivo de substâncias e os riscos associados pode incluir a educação de pares, através da formação no uso de substâncias, sobre os seus riscos e informação sobre serviços de saúde e sociais, isto quando o indivíduo não quer ou não consegue abandonar os consumos. Numa intervenção em rede focada no tratamento e reinserção, a questão que permanece é se a rutura com os membros da rede com CAD seria eficaz para promover a abstinência como muitos estudos sugerem. Será, assim, importante realizar uma análise mais aprofundada da rede social das pessoas com CAD, no sentido de compreender a interação e recurso aos membros da rede, assim como outros fatores de vulnerabilidade na rede e determinantes de processos excludentes.

Conflito de interesses | Conflict of interest: nenhum | none.

Fontes de financiamento | Funding sources: nenhuma | none.

\section{REFERÊNCIAS}

Alarcão, M. e Sousa, L. (2007). Rede social pessoal: do conceito à avaliação. Psychologica, 44, 353-376.

Andrade, P. , Carapinha, L., Sampaio, M., Shirley, S., Rodrigues, I., \& Silva, M. (2007). Para além do espelho a intervenção de proximidade nas toxicodependências. Toxicodependências, 13(2), 9-23.

Barbosa, J. (2011). Enfrentar "novos riscos" e resgatar a cidadania perdida: práticas de Serviço Social no seio das políticas de redução de danos. Toxicodependências, 17(1), 71-84.

Bohnert, A., Bradshaw, C. e Latkin, C. (2009). A social network perspective on heroin and cocaine use among adults: evidence of bidirectional influences. Addiction, 104, 1210-1218. doi: doi:10.1111/j.1360-0443.2009.02615.x.

Born, M. e Lionti, A.M. (1996). Familles pauvres et intervention en réseau. Paris: L'Harmattan.

Botella, H. C. (2007). Redes de Apoyo para la integración social: La família. Salud e drogas, 7(1), 45-56.

Castel, R. (2003 [1995]). As metamorfoses da questão social: uma crônica do salário ( $4 \cdot^{\text {a }}$ edição]. Pertópolis: Vozes.

Cavalcante, L., Falcão, R., Lima H., Marinho A., Macedo, J. e Braga, V. (2012). Rede de apoio social ao dependente químico: ecomapa como instrumental na assistência em saúde. Revista da Rede de Enfermagem do Nordeste 13(2),321-331. 
Chadi, M. (2000). Redes sociales en el trabajo social. Argentina: Espacio Editorial.

Costa, A. (2004). Exclusões sociais. Lisboa: Gradiva.

CRIA - Centro Respostas Integradas Aveiro (2012). Resumo de Diagnóstico Álcool e Drogas Concelho de Aveiro. Acedido em 15, março, 2015, In http://www.sicad.pt/BK/Concursos_v2/Documents/C_AV_010.pdf.

Davey, M., Latkin, C., Hua, W., Tobin, K. e Strathdee, S. (2007). Individual and social networks factors that predict entry to drug treatment. The American Jounal on Addictions, 16, 38-45.

El Bassel, N., Chen, D. e Cooper, D. (1998). Social Support and Social Network Profiles among Women on Methadone. Social Service Review, 72(3), 379-491.

EMCDDA (2002). Relatório anual sobre a evolução do fenómeno da droga na União Europeia e Noruega. Acedido em 13, outubro, 2016. In http://ar2002.emcdda.europa.eu/pt/page58-pt.html.

Fernandes, L., Pinto, M. e Oliveira, M. (2006). Caracterização e análise crítica das práticas de redução de riscos na área das drogas em Portugal. Toxicodependências 12(2), 71-82.

Garmendia, M., Alvarado, M., Montenegro, M. e Pino, P. (2008). Importancia del apoyo social en la permanência de la abstinência del consumo de drogas. Revista Médica Chile, 136, 169-178. DOI: doi:10.4067/S0034-98872008000200005.

Godinho, R. (2007). Estudo comparativo entre uma população toxicodependente sem-abrigo e uma população toxicodependente domiciliada aspetos psicossociais e psicopatológicos. Toxicodependências, 13(3), 3-14.

Guadalupe, S. (2009). Intervenção em rede: Serviço social, sistémica e redes de suporte social, Coimbra: Imprensa da Universidade de Coimbra. doi: http://sci-hub.cc/10.14195/978-989-26-0866-2.

Guay, J. (1984). L'intervenant professionnel face à l'aide naturelle. Montréal: Gaëtan Morin.

Lacroix, J. (1990). L'individu, sa famille et son réseau: les therápies familiales systémiques. Paris: ESF.

Leandro, M. e Ferreira, L. (2011). Os laços sociais em questão. Metamorfoses sociais, metamorfoses de uma nação. In M. Leandro (coord.). Laços familiares e sociais (pp. 27-57). Viseu: Psicossoma.

Ló, A. (2011). Integração social e estratégias de mediação. Toxicodependências, 17(1), 53-60.

López, S. A. (2010). Estrategias para la diversificación de la red personal de personas drogodependientes en processo de reinserción. REDES - Revista Hispana Para El Análisis de Redes Sociales, 18(7), 163-182.

Matias, M. e Fernandes, L. (2009). Desarrumar o medo...O arrumador de carros como figura do medo na cidade. Toxicodependências, 15(3), 9-22.

Mericle, A. (2014). The role of social networks in recovery from alcohol and drug abuse. The American Jornal of Drug and Alcohol Abuse, 40(3), 178-180. DOI: doi:10.3109/00952990.2013.875553.
Panebianco, D., Gallupe, O., Carrigton, P. e Colozzi, I. (2016). Personal support networks, social capital, and risk of relapse among individuals treated for substance use issues. International Jornal of Drug Policy, 27, 146-153. doi:10.1016/j.drugpo.2015.09.009.

Paugam, S. (2000). A desqualificação social. In M-H. Soulet (org.). Da não-integração (pp. 107-135). Coimbra: Quarteto.

Pinto, M. e Peixoto, S. (2003). Trabalhos de rua com consumidores de drogas - obstáculos sentidos no norte de Portugal. Toxicodependências, 9(2), 51-60.

Rosa, V. e Guadalupe, S. (2015). A rutura dos laços sociais nas narrativas da pessoa em situação de sem-abrigo. Intervenção Social, 42/45, 171-190.

SICAD - Serviço de Intervenção nos Comportamentos Aditivos e nas Dependências (2013). Relatório Anual 2013 - A situação do país em matéria de drogas e toxicodependências. Acedido em 12, dezembro, 2016. In http://www.sicad.pt/BK/Publicacoes/Lists/SICAD_PUBLICACOES/Attachm ents/72/Relat\%C3\%B3rioAnual_2013_A_Situa\%C3\%A7\%C3\%A30_do_Pa\%C3\% ADs_em_mat\%C3\%Agria_de_drogas_e_toxicodepend\%C3\%AAncias.pdf.

Silva, J. A. (2001). As redes sociais e o percurso na toxicodependência: o tratamento como Instrumento de Reinserção. Toxicodependências, 7(1), 23-34.

Sluzki, C. (1996). La red social: frontera de la pratica sistémica. Barcelona: Gedisa Editorial.

Soczka, L. e Nunes, J. (1989). Redes sociais de suporte e etiopatogenia do enfarte de miocárdio em meio urbano. Psicologia, VII(2), 157-166.

Souza, J, Kantorski L., Vasters, G. e Luís, M. (2011). Rede social de usuários de álcool, sob tratamento, em um serviço de saúde mental. Revista Latino-Americana Enfermagem, 19(1), 1-8. DOI: doi:10.1590/S0104-11692011000100019.

Souza, J. e Kantorski, L. (2009). A rede social de indivíduos sob tratamento em um CAPS ad: o ecomapa como recurso. Revista da Escola de Enfermagem USP, 43(2), 373-383. DOI: doi:10.1590/Soo80-62342009000200017.

Souza, J., Kantorski, L. e Mielke, F. (2006). Vínculos e redes sociais de indivíduos dependentes de substâncias psicoativas sob tratamento em CAPS AD. Revista Eletrónica Saúde Mental Álcool e Drogas, 2(1), 1-17.

Tracy, E., Min, M., Park, H., Jun, M., Brown, S. e Francis, M. (2016). Personal network structure and substance use in women by 12 months post treatment intake. Journal of Substance Abuse Treatment, 62, 55-61. doi: doi:10.1016/j.jsat.2015.11.002.

Tucker, J., Cheong, J., Chandler, S., Crawford, S. e Simpson, C. (2015) Social networks and substance use among at-risk emerging adults living in disadvantaged urban áreas in the Southern United States: a cross-sectional naturalistic study. Addiction, 110 1524-1532. doi: doi:10.1111/add.13010.

Vanegas, M.A.Z. (2009). La família, soporte para la recuperación de la adicción a las drogas. CES Psicología, 2(2), 86-94. 